\title{
Protective effect and molecular mechanism of liquiritin on oxybuprocaine-induced apoptosis of human corneal endothelial cells
}

\author{
$\mathrm{DAN} \mathrm{LI}^{1}$ and PENG ZHANG ${ }^{2}$ \\ Departments of ${ }^{1}$ Anesthesiology and ${ }^{2}$ Ophthalmology, \\ Ezhou Central Hospital of Wuhan University, Ezhou, Hubei 436000, P.R. China
}

Received August 25, 2017; Accepted January 31, 2018

DOI: $10.3892 /$ etm.2018.5860

\begin{abstract}
This study was designed to investigate the protective effect and possible molecular mechanism of liquiritin on oxybuprocaine-induced apoptosis of human corneal endothelial cells (HCECs). In this study, the effect of oxybuprocaine on the proliferation of HCEC-12 was detected using cell counting kit-8 (CCK-8). The inductive effect of oxybuprocaine on HCEC-12 apoptosis and protective effect of liquiritin against oxybuprocaine-induced HCEC-12 apoptosis were tested by Annexin V/propidium iodide (PI) staining and flow cytometry. The production of reactive oxygen species (ROS) was analyzed by 2,7-dichlorodi-hydrofluorescein diacetate (DCFH-DA) staining and fluorescent-activated cell sorting (FACS), and the expression of nuclear factor- $\mathrm{\kappa B}(\mathrm{NF}-\mathrm{\kappa B}) \mathrm{p} 65$ and apoptosis-related proteins, caspase- 3 and Bax, was determined by western blot analysis. Our results show that liquiritin resisted the inhibitory effect of oxybuprocaine on the proliferation of HCEC-12, and cell activity had the most significant increase in pretreatment with liquiritin group in the concentration of $8 \mathrm{mg} / \mathrm{ml}$; compared with that in oxybuprocaine group.Apoptosis in pretreatment with liquiritin was distinctly decreased and liquiritin resisted the production of ROS in HCEC-12 induced by oxybuprocaine. Investigation of molecular mechanism revealed that the pretreatment with liquiritin and pyrrolidinedithiocarbamic acid (PDTC) obviously blocked the expression of NF- $\mathrm{KB}$ p65 in nuclear protein increased by oxybuprocaine and the expression levels of total proteins, caspase- 3 and Bax.Moreover, tumor necrosis factor- $\alpha$ (TNF- $\alpha$ ) blocked the inhibitory effect of liquiritin on the expression of NF- $\mathrm{kB}$ p65 in nuclear protein and total proteins, caspase- 3 and Bax, thus
\end{abstract}

Correspondence to: Dr Peng Zhang, Department of Ophthalmology, Ezhou Central Hospital of Wuhan University, 9 Wenxing Road, Echeng, Ezhou, Hubei 436000, P.R. China

E-mail: xuanlizhang2004@163.com; 1839732942@qq.com

Key words: liquiritin, oxybuprocaine, human corneal endothelial cells, apoptosis, nuclear factor- $\kappa \mathrm{B}$ signal pathway obstructing the protective effect of liquiritin on corneal epithelial cells. The results of this study indicated that liquiritin reduces the expression of apoptosis protein and increases the expression of anti-apoptotic protein through inhibiting NF- $\mathrm{\kappa B}$ signal pathway, thus resisting HCEC-12 apoptosis induced by oxybuprocaine.

\section{Introduction}

Oxybuprocaine, as an ophthalmic topical anesthetic, is widely applied in a variety of eye surgery procedures. After drop administration with anesthetic, corneal sensation decreases or disappears and the epithelium loses its regularity and easily becomes dry, so it has certain toxic effects (1). One study (2) reported that allergic conjunctivitis and severe corneal damage have occurred after drop administration of oxybuprocaine after operation in clinical practice. Another study (3) revealed that $0.5-4 \mathrm{~g} / 1$ oxybuprocaine has an obvious inductive effect on apoptosis of human corneal endothelial cells (HCECs), displaying a significant concentration- and time-dependent manner, but the concentration of oxybuprocaine used in clinical practice is $4 \mathrm{~g} / \mathrm{l}$, so oxybuprocaine with clinical concentration has a strong inductive effect on apoptosis of HCECs. Thus, there is a need to develop efficient new drugs for HCECs to inhibit oxybuprocaine toxicity.

Licorice, belonging to Leguminosae Glycyrrhiza, is derived from the dry roots and rhizomes of Glycyrrhiza uralensis, Glycyrrhiza glabra and Glycyrrhiza inflata Batal., which is commonly added into a variety of traditional Chinese medicine compounds as an adjuvant or messenger drug; it tastes sweet and is neutral in nature, with effects such as invigorating spleen and replenishing $q i$, clearing away heat and toxic materials, expelling phlegm and arresting coughing, relieving spasm and stopping pain and moderating the property of herbs (1-3). Liquiritin is one of the main flavonoids in Glycyrrhiza uralensis, which has antidepressant, neuroprotective and therapeutic effects on heart system diseases (3-7). In Chinese traditional medicine, licorice has been used to treat eye disease, for example, viral keratitis, ulcerative keratitis, and irritability of keratitis. Liquiritin can significantly reduce apoptosis of human umbilical vein endothelial cells (HUVECs) induced by AGEs $(8,9)$ and play a strong protective effect on vascular endothelial cells in myocardial 
ischemia-reperfusion injury model $(6,10)$. It can also protect smoking-induced lung epithelial cell injury (11). Our past studies (unpublished)showed liquiritin was worthy of further study by HPLC-MS analysis and biological experiments. However, whether it can resist corneal epithelial cell damage by oxybuprocaine has not been reported yet.

This study investigated the protective effect of liquiritin on oxybuprocaine-induced apoptosis of HCECs, so as to provide some experimental foundation and theoretical basis for its application in clinical protection of corneal epithelial cells from injury.

\section{Materials and methods}

Cell culture. The HCEC-12 cells were purchased from Creative-Bioarray Co. (cat. no. CSC-C3457; New York, NY, USA) and placed in the RPMI-1640 medium containing 10\% fetal bovine serum (FBS) (both from HyClone, Logan, UT, USA), followed by placement in a cell culture incubator $\left(37^{\circ} \mathrm{C}\right.$, $5 \% \mathrm{CO}_{2}$ ). Penicillin and streptomycin with each concentration of $1.0 \times 10^{5} \mu \mathrm{l}$ were added into nutrient solution to resist bacterial contamination. Microscopic observation showed that cells were in the adherent growth in culture fluid, with multiplication every 26-48 h. The cell concentration was controlled at $10^{6}$ cells $/ \mathrm{ml}$. The fluid was changed every two days, and cells were subcultured once every four days. Oxybuprocaine: $0.4 \mathrm{~g}$ oxybuprocaine powder was dissolved in $100 \mathrm{ml}$ Dulbecco's modified Eagle's medium (DMEM)/F12 for preparation of $4 \mathrm{~g} / \mathrm{l}$ solution, adding medium to the desired concentration. Cells were cultured and oxybuprocaine was added into the cells on the second day for the required time.

Cell counting kit-8(CCK-8). The HCEC-12 in logarithmic growth phase was inoculated to the wells of a 96-well plate, followed by adjustment of density to $2 \times 10^{3}$ in each well. Subsequently, $200 \mu \mathrm{l}$ RPMI-1640 medium containing 10\% FBS was added. Six duplicated wells were set in each group. After culture for $24 \mathrm{~h}, 10 \mu \mathrm{l}$ CCK-8 solution was added into each well, and then the sample was incubated in an incubator containing $\mathrm{CO}_{2}$ for $4 \mathrm{~h}$. The well with phosphate-buffered solution (PBS) was regarded as the control, and the absorbance A value at $450 \mathrm{~nm}$ was detected by the enzyme analyzer. The growth curve was drawn.

Detection of apoptosis by flow cytometry. The adherent cells were digested with trypsin without ethylene diamine tetraacetic acid (EDTA) and collected (the digestion time was shortened as much as possible to avoid false positive); cells were rinsed by PBS twice (centrifuged at $600 \mathrm{x} \mathrm{g}$ for $5 \mathrm{~min}$ ), and then $1-5 \times 10^{5}$ cells were collected. Cell suspension $(500 \mu \mathrm{l})$ with binding buffer was added. After $5 \mu \mathrm{l}$ of Annexin V-family of intracellular (FITC) protein was added, followed by mixing well, then $5 \mu \mathrm{l}$ propidium iodide (PI) was added. The fluid was mixed well, and reacted at room temperature avoiding light for 5-15 min. The sample was observed and determined by flow cytometer within $1 \mathrm{~h}$ with excitation wavelength $\mathrm{Ex}=488 \mathrm{~nm}$ and emission wavelength Em=530 $\mathrm{nm}$. The green fluorescence of Annexin V was detected by FITC channel (FL1). The red fluorescence of PI was determined by PI channel using FL3. Statistical analysis was performed by GraphPad Software, Inc. (La Jolla, CA, USA).
Detection of reactive oxygen species (ROS). The treated cells were digested by pancreatin, followed by collection. The cells were re-suspended using pre-cooling PBS. Subsequently, serum-free medium was used to prepare $10 \mu \mathrm{M}$ probe dyeing working fluid. The pre-cooling and re-suspended cells were centrifuged and re-suspended in the probe dyeing working fluid, followed by mixing well to make the probe fully contact with cells. After incubation, cells were rinsed by serum-free medium three times, so as to fully remove 2,7-dichlorodi-hydrofluorescein diacetate (DCFH-DA) that did not enter the cells. The sample was detected by flow cytometry, followed by excitation with $480 \mathrm{~nm}$ wavelength and determination of emission light at $525 \mathrm{~nm}$. ROS-positive cells showed strong green fluorescence correspondening to FL1 detection channel of BD Biosciences (Franklin Lakes, NJ, USA) flow cytometer.

Western blotting. Polyacrylamide gel electrophoresis (PAGE) was conducted. The loading amount of protein in each well was $150 \mu \mathrm{g}$. Eighty volts was changed to $100 \mathrm{~V}$ for electrophoresis when Marker began to separate. When Marker was completely separated and the target band could be obtained, the electrophoresis was stopped. The protein was electrically transferred onto polyvinylidene fluoride (PVDF) membrane with electric current of $350 \mathrm{~mA}$ for $\sim 2 \mathrm{~h}$. The membrane was sealed with $5 \%$ bovine serum albumin (BSA)/milk at room temperature for $1 \mathrm{~h}$, followed by incubation with the diluted rabbit anti-human primary monoclonal antibodies $[\mathrm{NF}-\kappa \mathrm{B}$ p65 (cat. no. 4764), caspase-3 (cat. no. 9665), Bax (cat. no. 2774), B-cell lymphoma-2 (Bcl-2; cat. no. 2872) and glyceraldehyde3-phosphate dehydrogenase (GAPDH; cat. no. 2118); (all 1:1,000; Cell Signaling Technology, Inc., Danvers, MA, USA)] according to the instructions at $4^{\circ} \mathrm{C}$ overnight. On the second day, goat anti-rabbit secondary polyclpnal antibody (cat. no. 7074; 1:2,000; Cell Signaling Technology, Inc.) was added. Then, the sample was incubated at $37^{\circ} \mathrm{C}$ for $1 \mathrm{~h}$ and added with exposure liquid, followed by photographing using chemiluminescence apparatus.

Statistical analysis. Statistical results were analyzed by GraphPad Prism 5 software. The data are expressed as mean \pm standard deviation. The independent samples t-test was used for comparison of difference between two groups, and analysis of variance was adopted for comparison of multivariate means. $\mathrm{P}<0.05$ indicates that the difference was statistically significant.

\section{Results}

Oxybuprocaine inhibits the proliferation of HCEC-12, $H C E C-H 9 C 1$, and HCEC-B4G12. Oxybuprocaine is a common medicine for eye anesthesia. In order to study its influence on different human corneal endothelial cells, oxybuprocaine media with different concentrations were adopted to induce HCEC-12, HCEC-H9C1 and HCEC-B4G12 for 0, 3, 6,12 and $24 \mathrm{~h}$, and the effect of oxybuprocaine on activity of HCEC-12, HCEC-H9C1 and HCEC-B4G12 was detected by CCK-8. The results displayed that HCEC-12, HCEC-H9C1 and HCEC-B4G12 activity was inhibited and it was dependent on the concentration of oxybuprocaine; moreover, the cell activity of HCEC-12 was significantly inhibited after the reaction 

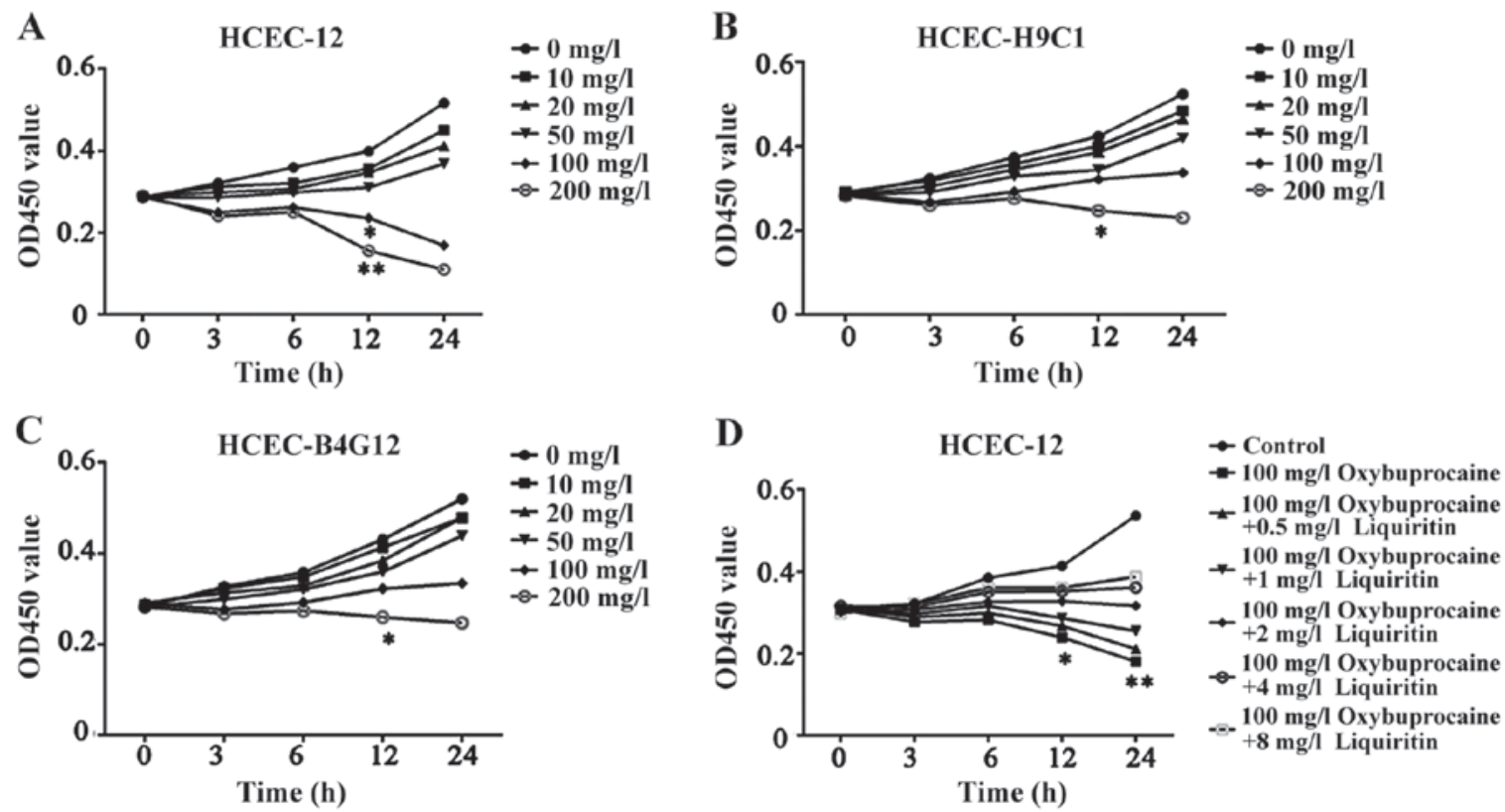

Figure 1. Detection of proliferation of HCEC-12, HCEC-H9C1, HCEC-B4G12 using CCK-8. (A-C) The effect of oxybuprocaine $(0,10,20,50,100$ and $200 \mathrm{mg} / \mathrm{l})$ on the proliferation of HCEC-12, HCEC-H9C1 and HCEC-B4G12 is detected using CCK-8. (D) Liquiritin $(0.5,1,2,4 \mathrm{and} 8 \mathrm{mg} / \mathrm{ml}) \mathrm{resisting}$ the inhibitory effect of oxybuprocaine on the proliferation of HCEC-12 was detected using CCK-8. ${ }^{*} \mathrm{P}<0.05$ and ${ }^{* *} \mathrm{P}<0.01$.

with concentration of oxybuprocaine over $100 \mathrm{mg} / \mathrm{l}$ for $12 \mathrm{~h}$ compared with those of HCEC-H9C1 and HCEC-B4G12 $(\mathrm{P}<0.05)$ (Fig. 1A-C). So we chose HCEC12 for the study.

Liquiritin resists the proliferation of HCEC-12 inhibited by oxybuprocaine. The high concentration of oxybuprocaine can induce HCEC-12 thus significantly reducing the activity of HCEC-12. In order to investigate the effect of liquiritin on proliferation of HCEC-12 induced by oxybuprocaine, HCEC-12 was pretreated by liquiritin in different concentrations, followed by being induced by $100 \mathrm{mg} / \mathrm{l}$ oxybuprocaine for $0,3,6,12$ and $24 \mathrm{~h}$. The activity of cells in each group was detected by CCK- 8 . The results showed that oxybuprocaine could significantly decrease cell activity compared with that in control group $(\mathrm{P}<0.05)$; the cell activity in pretreatment with liquiritin group was distinctly increased compared with that in oxybuprocaine group, and it showed the most significant increase in pretreatment with liquiritin group in the concentration of $8 \mathrm{mg} / \mathrm{ml}$ (Fig. 1D), indicating that liquiritin could resist the inhibitory effect of oxybuprocaine on the proliferation of HCEC-12.

Oxybuprocaine induces HCEC-12 apoptosis and ROS production. A variety of pro-apoptotic signals (such as unfavorable environmental factors, injury, radiation, chemotherapeutic agents, excitatory amino acid and death ligand) can cause increased cell endogenous or exogenous ROS or altered redox equilibrium. The production of ROS can serve as a signal triggering apoptosis in transduction pathway. Thus, HCEC-12 was intervened by oxybuprocaine media in different concentrations for $12 \mathrm{~h}$ in this study, and apoptosis was assessed by flow cytometry, revealing that compared with that in control group (1.9\%), $200 \mathrm{mg} / \mathrm{l}$ oxybuprocaine can significantly induce HCEC-12 apoptosis (20\%) (Fig. 2A and B). Additionally, DCFH-DA staining and fluorescent-activated cell sorting (FACS) were used to analyze the production of ROS after HCEC-12 was stimulated by oxybuprocaine, suggesting that different concentrations of oxybuprocaine could significantly induce the production of ROS in HCEC-12, which was concentration-dependent (Fig. 2C and D).

Liquiritin resists proliferation of HCEC-12 apoptosis and ROS production is induced by oxybuprocaine. In order to explore the effect of liquiritin on HCEC-12 apoptosis and ROS production induced by oxybuprocaine, HCEC-12 was pretreated by liquiritin in different concentrations for $1 \mathrm{~h}$, and then induced by $100 \mathrm{mg} / \mathrm{l}$ oxybuprocaine for $12 \mathrm{~h}$ in this study, and apoptosis and ROS production were detected by flow cytometry. The results revealed that compared with that in control group, apoptosis in oxybuprocaine group was distinctly increased, and it was remarkably reduced in pretreatment with liquiritin group compared with that in oxybuprocaine group (Fig. 3A and B). The production of ROS analyzed by DCFH-DA staining and FACS obtained results that were consistent with that of apoptosis, suggesting that liquiritin could resist the production of ROS in HCEC-12 induced by oxybuprocaine (Fig. 3C and D).

Liquiritin resists the $N F-\kappa B$ signal pathway activated by oxybuprocaine. Liquiritin can significantly reduce HCEC-12 apoptosis induced by oxybuprocaine thus protecting HCEC-12. It is well known that NF- $\mathrm{KB}$ signal pathway is widely involved in the process of apoptosis in many cells (12-15). Hence, this study aimed to investigate whether HCEC-12 apoptosis induced by oxybuprocaine is dependent on the NF- $\mathrm{KB}$ signal pathway, and whether liquiritin resists the induction of HCEC-12 apoptosis by oxybuprocaine through inhibiting the NF- $\kappa \mathrm{B}$ signal pathway. The results revealed that $50 \mathrm{mg} / \mathrm{l}$ oxybuprocaine could significantly increase the expression of NF- $\mathrm{KB}$ p 65 in nuclear protein (Fig. 4A) and decrease the expression of 

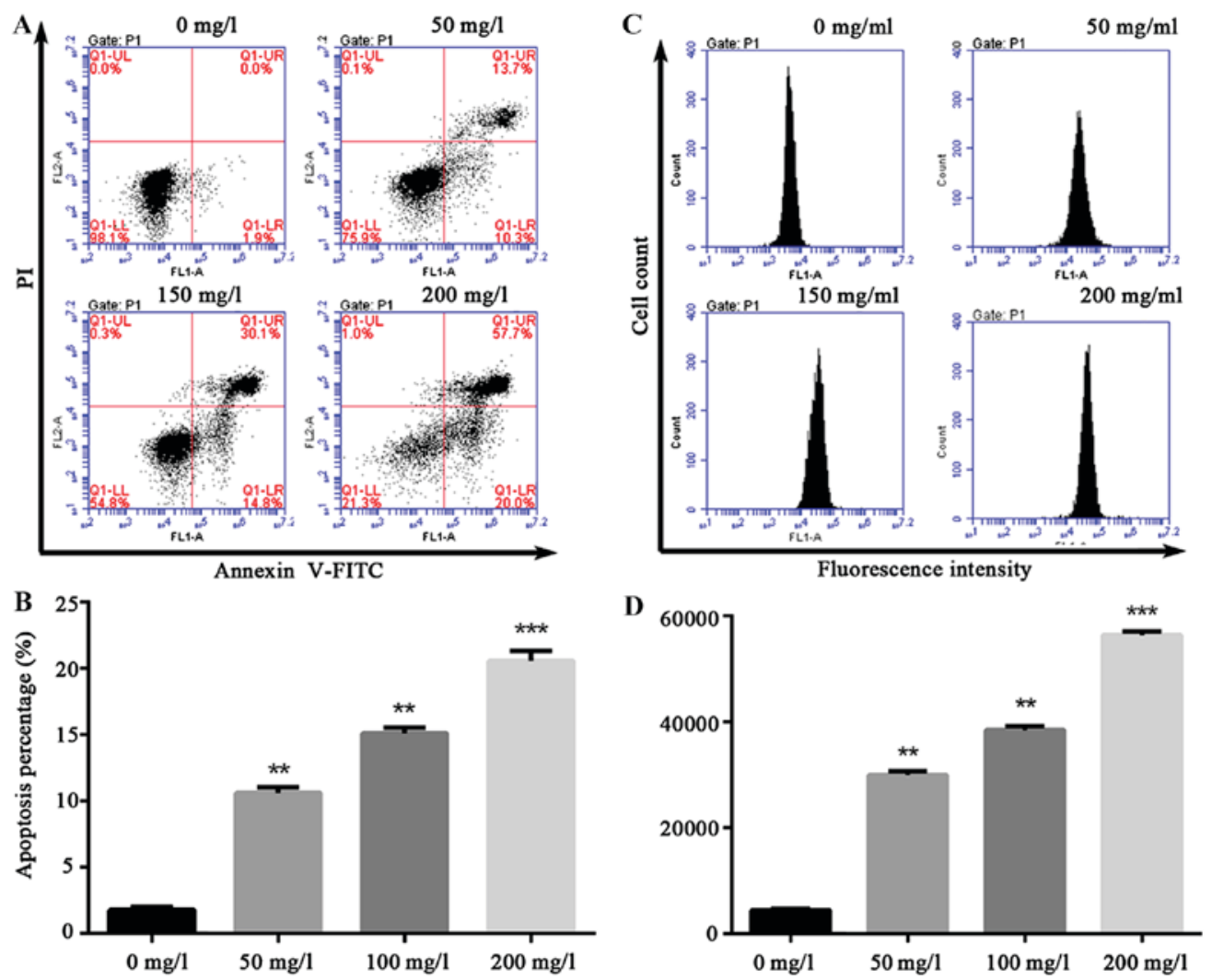

Figure 2. Effect of oxybuprocaine on HCEC-12 apoptosis and ROS production. (A and B) The effect of oxybuprocaine $(0,50,100 \mathrm{and} 200 \mathrm{mg} / \mathrm{l}) \mathrm{on} \mathrm{HCEC}-12$ apoptosis detected by flow cytometry. (B) Apoptosis percentage of early stage. (C and D) The effect of oxybuprocaine (0, 50, $100 \mathrm{and} 200 \mathrm{mg} / \mathrm{l}) \mathrm{on}$ ROS production in HCEC-12 cells analyzed by DCFH-DA staining and FACS. ${ }^{* *} \mathrm{P}<0.05$ and ${ }^{* * *} \mathrm{P}<0.01$.
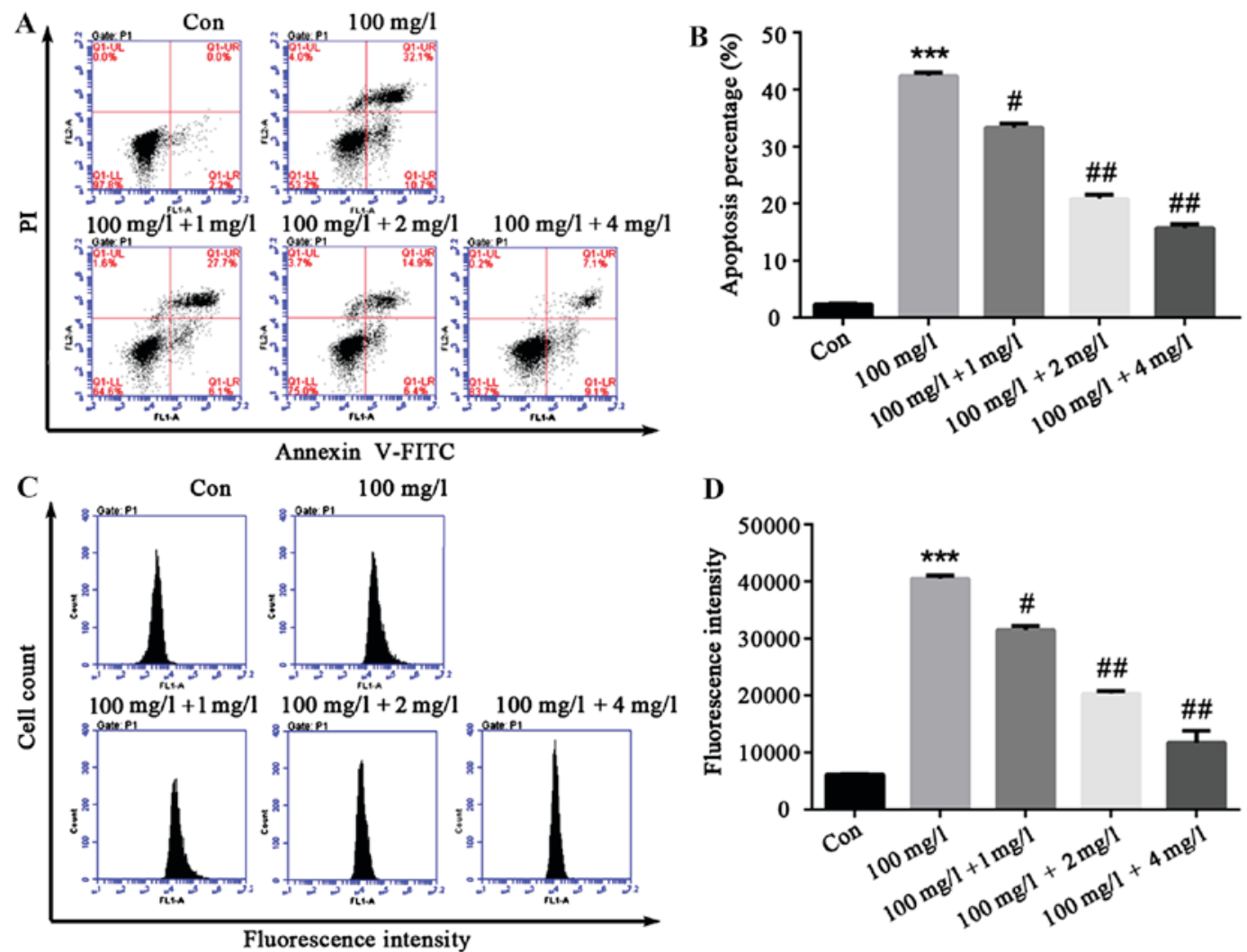

Figure 3. Effect of liquiritin on HCEC-12 apoptosis and ROS production induced by oxybuprocaine. (A and B) The effect of liquiritin (1,2 and $4 \mathrm{mg} / \mathrm{ml})$ on HCEC-12 apoptosis induced by oxybuprocaine was detected by flow cytometry. (B) Apoptosis percentage of early and late stage. (C and D) The effect of liquiritin $(1,2$ and $4 \mathrm{mg} / \mathrm{ml})$ on ROS production in HCEC-12 cells induced by oxybuprocaine was analyzed by DCFH-DA staining and FACS. ${ }^{* * *} \mathrm{P}<0.05$ compared with control group; ${ }^{\#} \mathrm{P}<0.05$ compared with oxybuprocaine group; ${ }^{\# /} \mathrm{P}<0.01$ compared with oxybuprocaine group. 
A

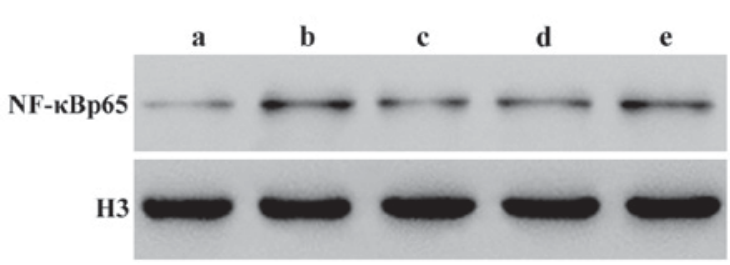

C

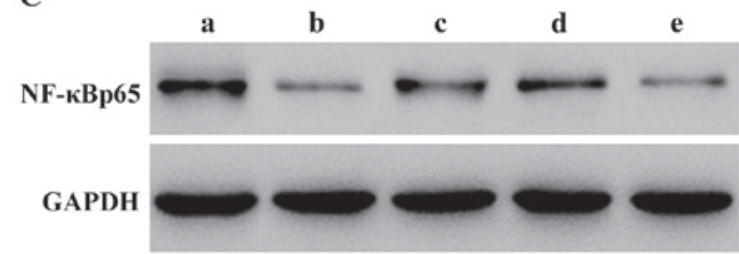

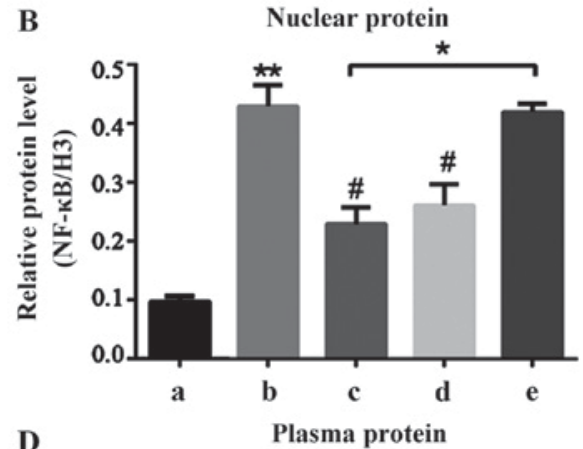

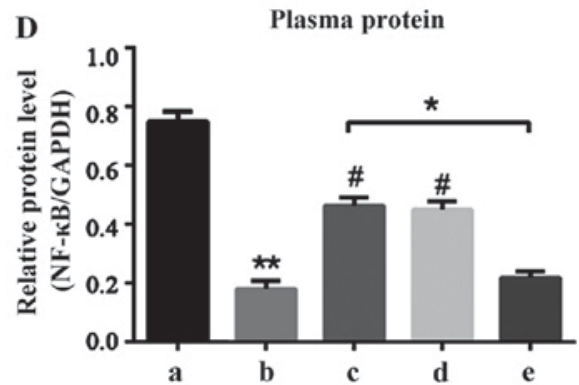

Figure 4. Liquiritin resistance of $\mathrm{NF}-\kappa \mathrm{B}$ signal pathway activated by oxybuprocaine. (A and B) The expression of NF- $\mathrm{B}$ p65 in nuclear protein of HCEC-12 cells is determined by western blotting. (C and D) The expression of NF- $\kappa \mathrm{B}$ p 65 in plasmosin in HCEC-12 cells is determined by western blotting. a, Control group. b, $50 \mathrm{mg} / \mathrm{l}$ oxybuprocaine group; c, oxybuprocaine $+2 \mathrm{mg} / \mathrm{l}$ liquiritin group; d, oxybuprocaine $+50 \mu \mathrm{mol} / 1 \mathrm{PTDC}$ group; e, oxybuprocaine $+2 \mathrm{mg} / 1$ liquiritin $+10 \mathrm{ng} / \mathrm{ml} \mathrm{TNF-} \alpha$ group. ${ }^{*} \mathrm{P}<0.05$ compared with oxybuprocaine $+2 \mathrm{mg} / 1$ liquiritin group; ${ }^{* *} \mathrm{P}<0.05$ compared with $50 \mathrm{mg} / 1$ oxybuprocaine group; ${ }^{\#} \mathrm{P}<0.05$ compared with control group.
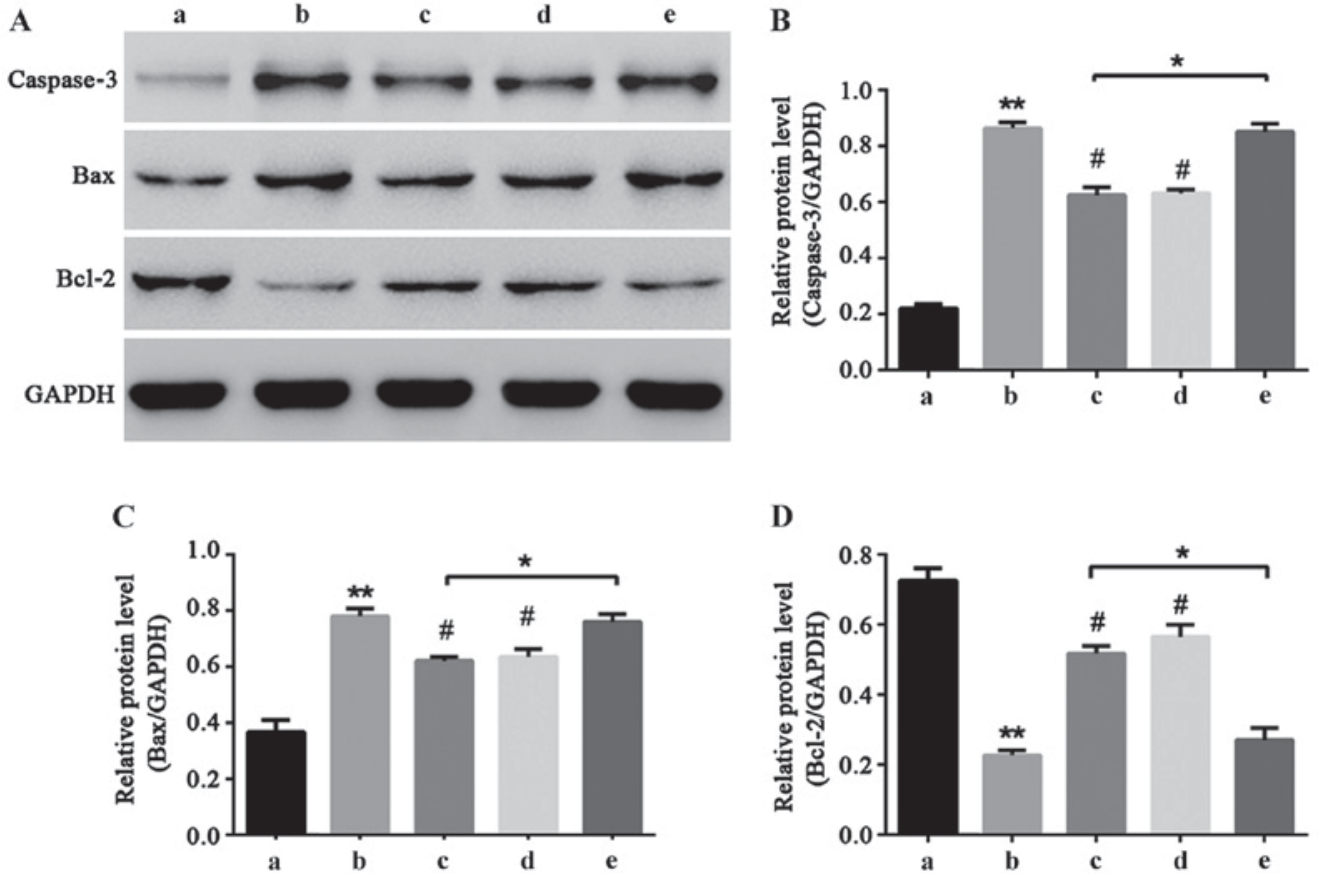

Figure 5. NF- $\kappa$ B signal pathway participates in liquiritin resistance of HCEC-12 apoptosis induced by oxybuprocaine. The expressions of caspase-3, Bax and $\mathrm{Bcl}-2$ proteins related to HCEC-12 apoptosis were determined by western blotting. a, Control group; b, $100 \mathrm{mg} / \mathrm{l}$ oxybuprocaine group; c, oxybuprocaine $+2 \mathrm{mg} / 1$ liquiritin group; d, oxybuprocaine $+50 \mu \mathrm{mol} / 1 \mathrm{PTDC}$ group; e, oxybuprocaine $+2 \mathrm{mg} / 1$ liquiritin $+10 \mathrm{ng} / \mathrm{ml} \mathrm{TNF}-\alpha$ group. ${ }^{*} \mathrm{P}<0.05 \mathrm{compared}$ with oxybuprocaine $+2 \mathrm{mg} / 1$ liquiritin group; ${ }^{* *} \mathrm{P}<0.05$ compared with $50 \mathrm{mg} / 1$ oxybuprocaine group; $\mathrm{P}<0.05$ compared with control group.

$\mathrm{NF}-\kappa \mathrm{B}$ p65 in plasmosin (Fig. 4B), and the pretreatment with $2 \mathrm{mg} / \mathrm{ml}$ liquiritin and $50 \mu \mathrm{mol} / 1$ pyrrolidinedithiocarbamic acid (PDTC) obviously blocked the expression of NF- $\kappa \mathrm{B}$ p65 in nuclear protein increased by oxybuprocaine (Fig. 4A). Additionally, $10 \mathrm{ng} / \mathrm{ml}$ tumor necrosis factor- $\alpha$ (TNF- $\alpha$ ) blocked the inhibitory effect of liquiritin on the expression of $\mathrm{NF}-\kappa \mathrm{B}$ p65 in nuclear protein (Fig. 4A), thus obstructing the protective effect of liquiritin, indicating that liquiritin resists HCEC-12 apoptosis induced by oxybuprocaine through inhibiting the $\mathrm{NF}-\kappa \mathrm{B}$ signal pathway.

$N F-\kappa B$ signal pathway participates in liquiritin resistance of HCEC-12 apoptosis induced by oxybuprocaine. Caspase-3, $\mathrm{Bax}$ and $\mathrm{Bcl}-2$ are important proteins that can directly reflect 
the extent of apoptosis (16-18). In order to explore the molecular mechanism of liquiritin resistance of HCEC-12 induced by oxybuprocaine, western blot was utilized to determine the expressions of caspase-3, Bax and Bcl-2 proteins. The results showed that oxybuprocaine increased the expression levels of caspase- 3 and Bax and reduced the expression levels of antiapoptotic protein $\mathrm{Bcl}-2$, and pretreatment with liquiritin and PDTC inhibited the increasing effect of oxybuprocaine on expression levels of caspase-3 and Bax proteins and reduced the inhibitory effect of oxybuprocaine on Bcl-2 expression level; pretreatment with TNF- $\alpha$ blocked the inhibitory effect of liquiritin on the expression levels of caspase-3 and Bax proteins and reduced the increasing effect of liquiritin on Bcl-2 expression (Fig. 5), revealing that liquiritin reduced the expression of apoptosis proteins and increased the expression of anti-apoptosis proteins through inhibiting the NF- $\kappa \mathrm{B}$ signal pathway, thus resisting HCEC-12 apoptosis induced by oxybuprocaine.

\section{Discussion}

With the development of ophthalmic surgery techniques and equipment, ophthalmic topical anesthetics have been widely applied, and its side effects on cornea are getting increasing attention. The most commonly used topical anesthetic is oxybuprocaine (8-10). Cornea is mainly composed of endothelium, stroma, epithelium and its derivatives, and corneal endothelial cells in mammals (except for rabbits) lose their regenerative ability in adulthood. Therefore, the damage of human corneal endothelial cells cannot be repaired, and the study on HCEC is of uppermost priority. Previous studies show that oxybuprocaine can induce apoptosis of corneal epithelial cells, which causes certain damage on corneal epithelial cells, so it is urgent to develop drugs that can resist toxicity of oxybuprocaine (15-18).

Liquiritin is derived from Glycyrrhiza uralensis which belongs to leguminous plants. It has good anti-inflammatory and antioxidant activity (18). However, its protective effect on corneal epithelial cell injury has not been reported. The experimental results showed that oxybuprocaine inhibited the proliferation of human corneal epithelial cells and induced its apoptosis, which was concentration-dependent. The results of pretreatment with liquiritin revealed that oxybuprocaine significantly decreased cell activity compared with that in control group $(\mathrm{P}<0.05)$; the cell activity in pretreatment with liquiritin group was distinctly increased compared with that in oxybuprocaine group, and it showed the most significant increase in pretreatment with liquiritin group in the concentration of $8 \mathrm{mg}$ / $\mathrm{ml}$, indicating that liquiritin could resist the inhibitory effect of oxybuprocaine on the proliferation of HCEC-12. The results of apoptosis experiment showed that compared with that in the control group, apoptosis in oxybuprocaine group was distinctly increased, and it was remarkably reduced in pretreatment with liquiritin group compared with that in oxybuprocaine group. The production of ROS analyzed by DCFH-DA staining and FACS obtained results which were consistent with that of apoptosis, suggesting that liquiritin can resist the production of ROS in HCEC-12 induced by oxybuprocaine.

The results of molecular mechanism investigation revealed that oxybuprocaine significantly increased the expression of
$\mathrm{NF}-\kappa \mathrm{B}$ p65 in nuclear protein and decreased NF- $\kappa \mathrm{B}$ p65 in plasmosin, and the pretreatment with liquiritin and PDTC obviously blocked the expression of NF- $\mathrm{NB}$ p65 in nuclear protein increased by oxybuprocaine. Additionally, TNF- $\alpha$ blocked the inhibitory effect of liquiritin on the expression of $\mathrm{NF}-\kappa \mathrm{B}$ p65 in nuclear protein, thus obstructing the protective effect of liquiritin. The results of western blotting showed that oxybuprocaine increased the expression levels of caspase- 3 and Bax and reduced the expression levels of anti-apoptotic protein Bcl-2, and pretreatment with liquiritin and PDTC inhibited the increasing effect of oxybuprocaine on expression levels of caspase- 3 and Bax proteins and reduced the inhibitory effect of oxybuprocaine on Bcl-2 expression level; pretreatment with TNF- $\alpha$ blocked the inhibitory effect of liquiritin on the expression levels of caspase- 3 and Bax proteins. NF- $\kappa \mathrm{B}$ has been reported to inhibit apoptosis or promote apoptosis depending on the contexts (19). The downregulation of Bcl-2, upregulation of Bax, and activation of caspase- 3 are widely known in the occurrence of apoptosis. We found that oxybuprocaine induced changes of protein levels of $\mathrm{NF}-\kappa \mathrm{B}, \mathrm{Bcl}-2$, Bax and caspase-3, but did not further analyze the relationship between NF- $\mathrm{B}$ and $\mathrm{Bcl}-2$, Bax, or caspase-3. Regarding the relationship between $\mathrm{NF}-\kappa \mathrm{B}$ and $\mathrm{Bcl}-2$, Bax, or caspase-3, Wier et al (20) showed that despite the cleavage of NF- $\mathrm{KB}$ p65 by caspase- 3 , the cleavage-generated p $65 \mathrm{~N}$-terminal fragment interferes with the RPS3/NF- $\mathrm{B}$-confering gene transcription. Cao et al (21) also found that inhibition of NF- $\kappa \mathrm{B}$ lead to increase of Bcl-2 expression and attenuates caspase-3 activation. Therefore, there were interactions between $\mathrm{NF}-\kappa \mathrm{B}$ and $\mathrm{Bcl}-2$, Bax or caspase-3, but the detailed relationships in the context of liquiritin against oxybuprocaine-induced apoptosis need to be analyzed in further studies.

In conclusion, the results of this study indicated that liquiritin reduces the expression of apoptosis proteins and increases the expression of anti-apoptosis proteins through inhibiting NF- $\mathrm{NB}$ signal pathway, thus resisting HCEC-12 apoptosis induced by oxybuprocaine. The protective effect of liquiritin on corneal epithelial cells is expected to be used in the clinical practice to inhibit the toxicity of oxybuprocaine on corneal epithelial cells.

\section{Acknowledgements}

Not applicable.

\section{Funding}

No funding was received.

\section{Availability of data and materials}

The datasets used and/or analyzed during the present study are available from the corresponding author on reasonable request.

\section{Authors' contributions}

DL collected, analyzed and interpreted the patient data, and drafted the manuscript. PZ conceived and designed the study, and revised the manuscript for important intellectual content. Both authors read and approved the final manuscript. 


\section{Ethics approval and consent to participate}

Not applicable.

\section{Consent for publication}

Not applicable.

\section{Competing interests}

The authors declare that they have no competing interests.

\section{References}

1. Hatano T, Yasuhara T, Miyamoto K and Okuda T: Anti-human immunodeficiency virus phenolics from licorice. Chem Pharm Bull (Tokyo) 36: 2286-2288, 1988.

2. Kelly-Pieper K, Patil SP, Busse P, Yang N, Sampson H, Li XM, Wisnivesky JP and Kattan M: Safety and tolerability of an antiasthma herbal Formula (ASHMI) in adult subjects with asthma: A randomized, double-blinded, placebo-controlled, dose-escalation phase I study. J Altern Complement Med 15: 735-743, 2009.

3. Whorwood CB, Sheppard MC and Stewart PM: Licorice inhibits 11 beta-hydroxysteroid dehydrogenase messenger ribonucleic acid levels and potentiates glucocorticoid hormone action. Endocrinology 132: 2287-2292, 1993.

4. Tamir S, Eizenberg M, Somjen D, Stern N, Shelach R, Kaye A and Vaya J: Estrogenic and antiproliferative properties of glabridin from licorice in human breast cancer cells. Cancer Res 60: 5704-5709, 2000.

5. Hatano T, Shintani Y, Aga Y, Shiota S, Tsuchiya T and Yoshida T: Phenolic constituents of licorice. VIII. Structures of glicophenone and glicoisoflavanone, and effects of licorice phenolics on methicillin-resistant Staphylococcus aureus. Chem Pharm Bull (Tokyo) 48: 1286-1292,2000.

6. Sun YX, Tang Y, Wu AL, Liu T, Dai XL, Zheng QS and Wang ZB: Neuroprotective effect of liquiritin against focal cerebral ischemia/reperfusion in mice via its antioxidant and antiapoptosis properties. J Asian Nat Prod Res 12: 1051-1060, 2010.

7. Takahashi T, Takasuka N, Iigo M, Baba M, Nishino H, Tsuda $H$ and Okuyama T: Isoliquiritigenin, a flavonoid from licorice, reduces prostaglandin E2 and nitric oxide, causes apoptosis, and suppresses aberrant crypt foci development. Cancer Sci 95: 448-453, 2004

8. Zhang X, Song Y, Han X, Feng L, Wang R, Zhang M, Zhu M, Jia $X$ and $\mathrm{Hu}$ S: Liquiritin attenuates advanced glycation end products-induced endothelial dysfunction via RAGE/NF- $\kappa \mathrm{B}$ pathway in human umbilical vein endothelial cells. Mol Cell Biochem 374: 191-201, 2013.

9. Feng L, Zhu MM, Zhang MH, Wang RS, Tan XB, Song J, Ding SM, Jia XB and Hu SY: Protection of glycyrrhizic acid against AGEs-induced endothelial dysfunction through inhibiting RAGE/NF- $\kappa$ B pathway activation in human umbilical vein endothelial cells. J Ethnopharmacol 148: 27-36, 2013.
10. Guoqiang Q and Guoping Z: Optimal proportion of four effective components of Danggui Decoction on vascular endothelial cell protection in rats with myocardial ischemia reperfusion injury. Tradit Chin Med Mater 34: 580-584, 2011 (In Chinese).

11. Guan Y, Li FF, Hong L, Yan XF, Tan GL, He JS, Dong XW, Bao MJ and Xie QM: Protective effects of liquiritin apioside on cigarette smoke-induced lung epithelial cell injury. Fundam Clin Pharmacol 26: 473-483, 2012.

12. Koshimizu JY, Beltrame FL, de Pizzol JP Jr, Cerri PS, Caneguim BH and Sasso-Cerri E: NF- $\kappa$ B overexpression and decreased immunoexpression of $\mathrm{AR}$ in the muscular layer is related to structural damages and apoptosis in cimetidine-treated rat vas deferens. Reprod Biol Endocrinol 11: 29, 2013

13. Arora R, Yates C, Gary BD, McClellan S, Tan M, Xi Y, Reed E, Piazza GA, Owen LB and Dean-Colomb W: Panepoxydone targets $\mathrm{NF}-\kappa \mathrm{B}$ and $\mathrm{FOXM} 1$ to inhibit proliferation, induce apoptosis and reverse epithelial to mesenchymal transition in breast cancer. PLoS One 9: e98370, 2014.

14. Decean H, Fischer-Fodor E, Tatomir C, Perde-Schrepler M, Somfelean L, Burz C, Hodor T, Orasan R and Virag P: Vitis vinifera seeds extract for the modulation of cytosolic factors BAX- $\alpha$ and NF- $\kappa$ B involved in UVB-induced oxidative stress and apoptosis of human skin cells. Clujul Med 89: 72-81, 2016.

15. Zuo N, Zheng X, Liu H and Ma X: Fenofibrate, a PPAR $\alpha$ agonist, protect proximal tubular cells from albumin-bound fatty acids induced apoptosis via the activation of $\mathrm{NF}-\kappa \mathrm{B}$. Int J Clin Exp Pathol 8: 10653-10661, 2015.

16. Nicholson DW and Thornberry NA: Caspases: Killer proteases. Trends Biochem Sci 22: 299-306, 1997.

17. Shi L, Teng H, Zhu M, Li C, Huang K, Chen BI, Dai Y and Wang J: Paeoniflorin inhibits nucleus pulposus cell apoptosis by regulating the expression of $\mathrm{Bcl}-2$ family proteins and caspase- 9 in a rabbit model of intervertebral disc degeneration. Exp Ther Med 10: 257-262, 2015.

18. Liu Z, Ding Y, Ye N, Wild C, Chen H and Zhou J: Direct activation of bax protein for cancer therapy. Med Res Rev 36: 313-341, 2016.

19. Jing $\mathrm{H}$ and Lee $\mathrm{S}: \mathrm{NF}-\kappa \mathrm{B}$ in cellular senescence and cancer treatment. Mol Cells 37: 189-195, 2014.

20. Wier EM, Fu K, Hodgson A, Sun X and Wan F: Caspase-3 cleaved p65 fragment dampens NF- $\kappa \mathrm{B}$-mediated anti-apoptotic transcription by interfering with the p65/RPS3 interaction. FEBS Lett 589: 3581-3587, 2015.

21. Cao ZH, Yin WD, Zheng QY, Feng SL, Xu GL and Zhang KQ: Caspase- 3 is involved in IFN- $\gamma$ - and TNF- $\alpha$-mediated MIN6 cells apoptosis via NF-кB/Bcl-2 pathway. Cell Biochem Biophys 67: $1239-1248,2013$

This work is licensed under a Creative Common Attribution-NonCommercial-NoDerivatives 4.0 International (CC BY-NC-ND 4.0) License. 